\title{
Photonic band structures of 2D non-circular air lattices in $\mathrm{Si}$
}

\author{
R. Hillebrand ${ }^{\mathrm{a}, *}, \mathrm{~W}$. Hergert ${ }^{\mathrm{b}}, \mathrm{W}$. Harms $^{\mathrm{a}}$ \\ ${ }^{a}$ Max Planck Institute of Microstructure Physics, Weinberg 2, D-06120 Halle, Germany \\ ${ }^{\mathrm{b}}$ Department of Physics, MLU Halle, Friedemann-Bach-Platz 6, D-06108 Halle, Germany
}

\begin{abstract}
In recent years, the fabrication and physical characterization of photonic crystals have been met with increasing interest. In our contribution, theoretical dispersion studies of Si-based photonic crystals are presented. Solving Maxwell's equations by 'plane wave expansions', band structures and densities of states are computed for triangular 2D lattices of air columns in silicon. A step-by-step variation of the pore size provides batches of band structures combined to comprehensive gap maps. Main concern of the paper are investigations of band gap modifications (TM and TE polarization) for pore shapes of deteriorating roundness, approximated by ellipses of varying eccentricity. (C) 2001 Elsevier Science Ltd. All rights reserved.
\end{abstract}

Keywords: Photonic crystal; Silicon; Band structure; Density of states; Gap map

\section{Introduction}

The extraordinary optical properties of artificial photonic crystals have been described by several authors (see, e.g., [1,2]) in the last decade. As a promising candidate for manufacturing photonic crystals, silicon combines a well-established technology with an appropriately high dielectric constant. The practical aim of the studies presented is to support the interpretation of IR measurements of macroporous photonic band gap materials $[3,4]$ that are electro-chemically prepared.

From the point of view of theoretical physics, photonic crystals are described by their band structures (PBS), the density of states (DOS) and the transmission spectrum. Routinely, 2D lattices of triangular or square types are assumed to consist of columns (holes or rods) of circular shape. The dispersion behaviour of the TM and TE modes can be controlled by the lattice filling factor and the dielectric contrast between the columns and the background.

\footnotetext{
*Corresponding author. Tel.: +49-345-5582911; fax: +49345-5511223.

E-mail address: hi@mpi-msp-halle.mpg.de (R. Hillebrand).
}

The quality of the etched lattices of air columns in silicon, especially the size distribution and the roundness of the holes, has been inspected by SEM image processing. It turned out that there are significant deviations from the circular shape of the pores due to the crystalline structure of the $\mathrm{Si}$ substrate and the square $\mathrm{KOH}$ etch pits, used for the pore formation process [5]. In the present paper, analytical formulas and numerical results are presented to study the influence of deteriorating structural roundness on the PBS and DOS data. The geometrical character of the single 'atoms' of the lattice has been transformed to ellipses of varying eccentricity. The questions theoretically relevant are

(i) In which way do the band gaps, especially the complete gaps, depend upon the eccentricity of air columns?

(ii) Is it possible to increase the size of band gaps?

(iii) Is there any correlation between the orientation of the ellipses and the gap size in definite k-directions (wave guide properties)?

In the next section the theoretical background of the studies will be briefly described, followed by the discussion of the results and conclusions. 


\section{Theory}

The dielectric material is assumed to be isotropic having structural details on a macroscopic scale. The relative permeability $\mu$ is 1 . The dielectric constant is therefore assumed to be scalar and independent of the frequency of the light. Because of the two-dimensional lattice periodicity, the dielectric constant $\varepsilon$ is described by

$\varepsilon(\mathbf{r})=\varepsilon(\mathbf{r}+\mathbf{R})$.

The vectors $\mathbf{R}$ are vectors of the $2 \mathrm{D}$ lattice. Solving Maxwell's equations for the magnetic field $\mathbf{H}_{\omega}$ yields the so-called "master equation" (cf., e.g., [6-8]).

$\nabla \times\left(\frac{1}{\varepsilon(\mathbf{r})} \nabla \times \mathbf{H}_{\omega}(\mathbf{r})\right)=\left(\frac{\omega}{c}\right)^{2} \mathbf{H}_{\omega}(\mathbf{r})$.

where $\omega$ is the frequency of the light, and $c$ the light velocity.

The magnetic field $\mathbf{H}_{\boldsymbol{\omega}}$ is expanded into plane waves of wave vector $\mathbf{k}$ with respect to the two-dimensional reciprocal lattice vectors $\mathbf{G}$ (for alternative approaches see, e.g., $[9,10])$ :

$\mathbf{H}_{\omega}(\mathbf{r})=\sum_{\mathbf{G} \lambda} h_{\mathbf{G} \lambda} \hat{\mathbf{e}}_{\lambda} \mathrm{e}^{[\mathrm{i}(\mathbf{k}+\mathbf{G}) \cdot \mathbf{r}]}$.

Two independent polarizations $\lambda$ characterized by the polarization vectors $\hat{\mathbf{e}}_{\lambda}$ are considered. Using Eq. (3) in the master equation (2) provides an equation for the coefficients $h_{\mathbf{G} \lambda}$

$\sum_{\mathbf{G}^{\prime} \lambda^{\prime}} \Xi_{\mathbf{G} \lambda, \mathbf{G}^{\prime} \lambda^{\prime}}^{\mathbf{k}} h_{\mathbf{G}^{\prime} \lambda^{\prime}}=\omega^{2} h_{\mathbf{G} \lambda}$.

The matrix $\Xi$ in the eigenvalue equation is defined by

$\Xi_{\mathbf{G} \lambda, \mathbf{G}^{\prime} \lambda^{\prime}}^{\mathbf{k}}=\left[(\mathbf{k}+\mathbf{G}) \times \hat{\mathbf{e}}_{\lambda}\right]\left[\left(\mathbf{k}+\mathbf{G}^{\prime}\right) \times \hat{\mathbf{e}}_{\lambda^{\prime}}\right] \varepsilon^{-1}\left(\mathbf{G}, \mathbf{G}^{\prime}\right)$.

The Fourier transform of the inverse dielectric constant $\varepsilon^{-1}\left(\mathbf{G}, \mathbf{G}^{\prime}\right)=\varepsilon^{-1}\left(\mathbf{G}-\mathbf{G}^{\prime}\right)$ depends on the difference of the reciprocal lattice vectors, only. The properties of $\varepsilon(\mathbf{r})$ in the unit cell ( $A$ is the area of the unit cell)

$\varepsilon^{-1}(\mathbf{G})=\frac{1}{A} \int_{A} \varepsilon^{-1}(\mathbf{r}) \mathrm{e}^{-\mathrm{i} \mathbf{G r}} \mathrm{d}^{2} \mathbf{r}$

as well as the lattice type and the polarization of the photon modes determine the photonic band structure. For 2D crystals and in-plane propagation, the eigenmodes of the vector field decouple to modes of the electric field vector being parallel or perpendicular to the pore axes, called TM and TE polarization, respectively. A large number of plane waves, typically up to 500, are necessary to attain convergence, if the dielectric contrast is high. Problems of convergence are discussed, for instance, in [10,11].

To investigate the influence of the eccentricity of the air columns on PBS and DOS, analytical solutions have been derived for the Fourier transform of $\varepsilon^{-1}$ for elliptical columns (cf. Fig. 1). The model geometry in

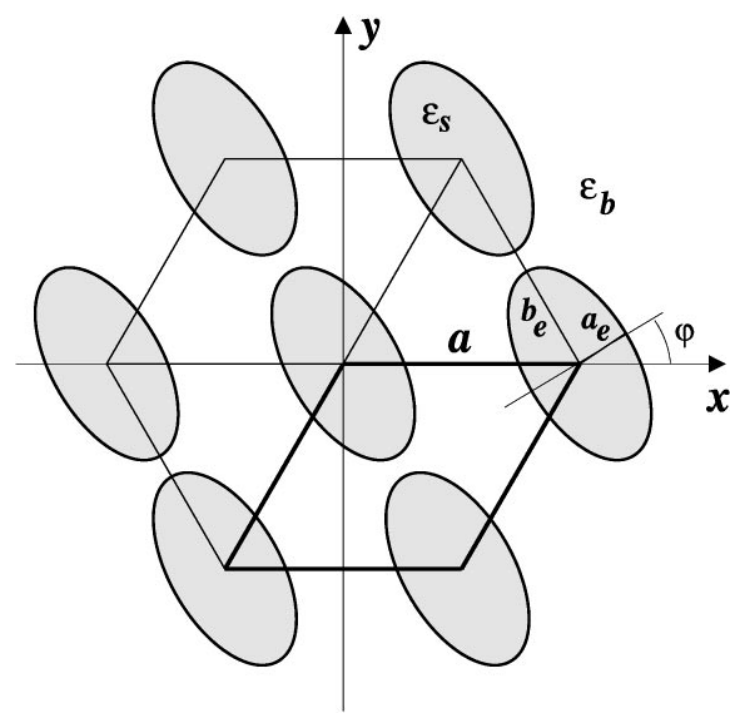

Fig. 1. Model of a 2D photonic crystal (triangular lattice of elliptical pores).

real space is described by

$\varepsilon(\mathbf{r})=\varepsilon_{\mathrm{b}}+\left(\varepsilon_{\mathrm{s}}-\varepsilon_{\mathrm{b}}\right) f(\mathbf{r})$.

( $f(\mathbf{r})$ equals 1 inside the column, and 0 outside of it.)

The quantity $\varepsilon_{\mathrm{S}}$ is the dielectric constant of the columns, and $\varepsilon_{\mathrm{b}}$ is the dielectric constant of the background, respectively. The elliptical columns may show different orientations with respect to the lattice. The dimensions of the rods are given by the major and minor axes $\left(b_{e}, a_{e}\right)$ resulting in an eccentricity of $e=a_{e} / b_{e}$. The Fourier transform $\varepsilon^{-1}(\mathbf{G})$ for the real structure of Fig. 1 reads

$\varepsilon^{-1}(\mathbf{G})=\frac{1}{\varepsilon_{\mathrm{b}}} \delta_{\mathbf{G}, 0}+\left(\frac{1}{\varepsilon_{\mathrm{s}}}-\frac{1}{\varepsilon_{\mathrm{b}}}\right) \gamma_{e} \frac{2 J_{1}\left(a_{e} \sqrt{g(\varphi)}\right)}{a_{e} \sqrt{g(\varphi)}}$.

The factor $\gamma_{e}=2 \pi a_{e} b_{e} /\left(a^{2} \sqrt{3)}\right.$ stands for the 'filling factor', describing the area parts of the column and the background. Function $g(\varphi)$ describes the orientation of the column in the unit cell:

$$
\begin{aligned}
g(\varphi)= & G_{x}^{2}\left(\cos ^{2} \varphi+\left(\frac{b_{e}}{a_{e}}\right) \sin ^{2} \varphi\right) \\
& +G_{y}^{2}\left(\sin ^{2} \varphi+\left(\frac{b_{e}}{a_{e}}\right) \cos ^{2} \varphi\right) \\
& +2 G_{x} G_{y} \cos \varphi \sin \varphi\left(1-\left(\frac{b_{e}}{a_{e}}\right)\right) .
\end{aligned}
$$

A rotation of the rod by $\varphi=\pi / 2$ corresponds to a change of the major and minor axes. For a rod being circular, i.e. $a_{e}=b_{e}, g(\varphi)=G$. 


\section{Results}

In general, the dispersion properties of $2 \mathrm{D}$ photonic crystals are controlled by the dielectric contrast, the lattice type, i.e. square or triangular geometry, and the filling ratio, experimentally scaled by the lattice parameter $a$. Fig. 2 shows the variations of the band structures calculated for perfectly circular air columns in silicon $(\varepsilon=11.7)$, arranged in a triangular lattice, in the experimentally relevant $r / a$ region. The $r / a$ ratio, i.e. the diameter of the air holes, increases linearly from left to right. For each filling factor, the TM and the TE modes are plotted following the $\mathbf{k}$-path $\mathbf{\Gamma}-\mathbf{M}-\mathbf{K}-\mathbf{\Gamma}$ in the Brillouin zone. The variations of the complete band gaps can be observed in the horizontal direction. The complete gap is maximum near $r / a=0.475$, reaching the value $\Delta f=0.152[\omega a / \pi c]$.

As Fig. 2 indicates, the band gaps of the TM and TE modes pass each other, with the midgap frequencies of all gaps increasing with increasing air filling ratio.

The plots of the density of states of a photonic crystal with an $r / a$ ratio of 0.458 are shown in Fig. 3, revealing that the 'forbidden frequencies' around $f \in[0.9,1.0]$

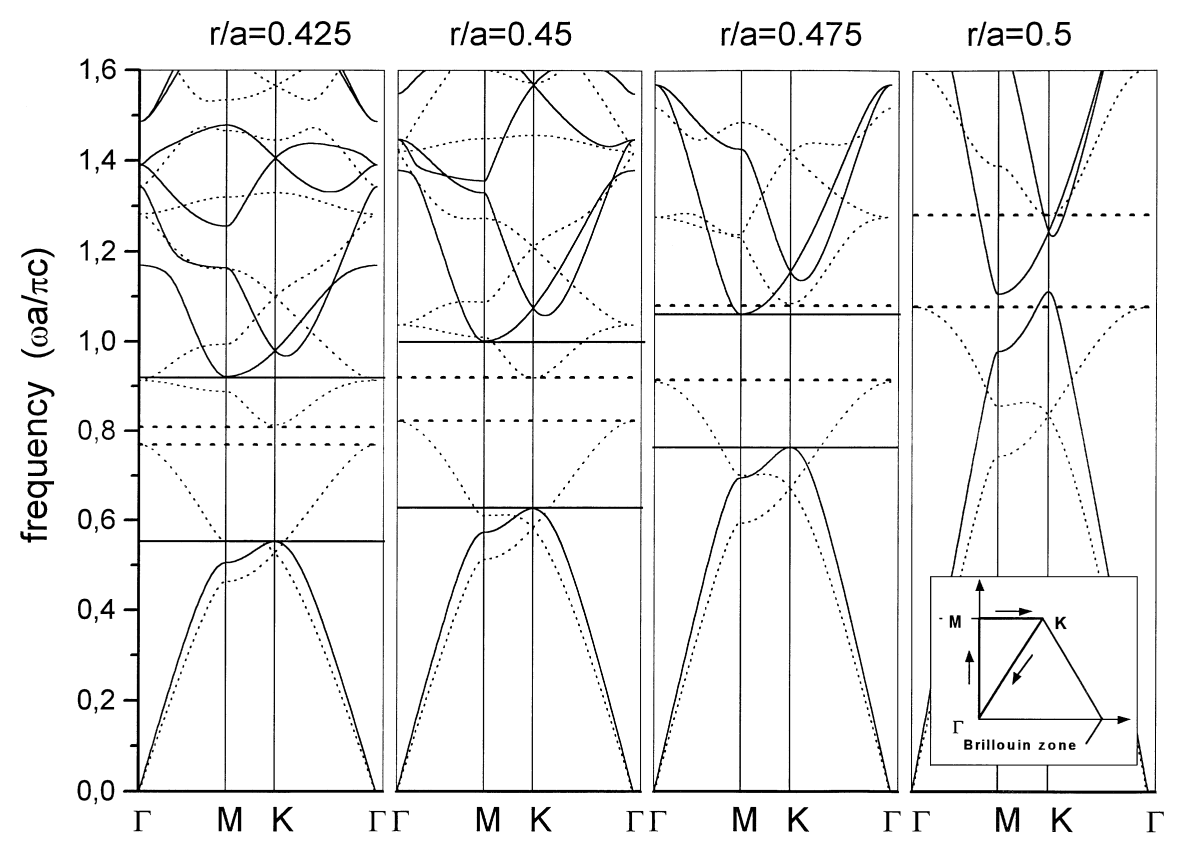

Fig. 2. TM bands (dotted lines) and TE bands (solid lines), calculated for a triangular lattice of circular holes in $\mathrm{Si}(\varepsilon=11.7)$, varying the ratio $r / a$. The gaps are marked by lines (967 waves).

TM mode

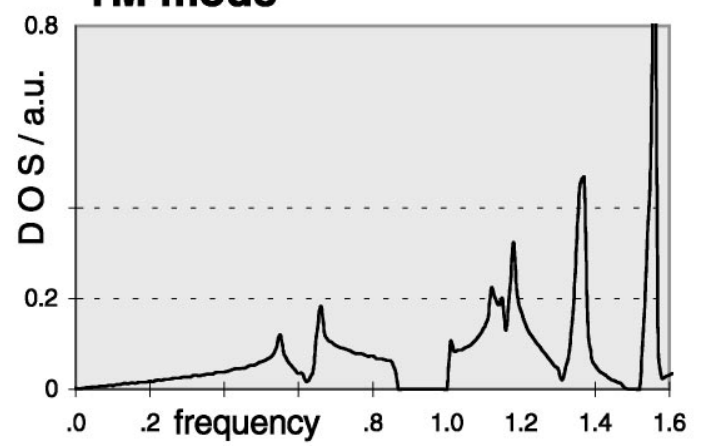

\section{TE mode}

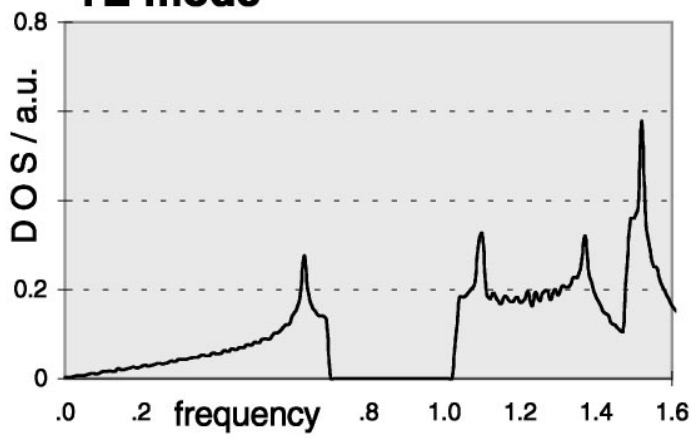

Fig. 3. Calculated density of states (DOS, TM and TE modes) of a triangular air lattice in Si $(f[\omega a / \pi c], \varepsilon=11.7, r / a=0.458$, 5151 k-points, 271 waves). 
form a complete photonic band gap. For both, rapidly decreasing and increasing air filling ratios $r / a$, the forbidden states $(\mathrm{DOS}=0)$, occurring for the $\mathrm{TM}$ as well as for the TE polarization, do no longer correspond with each other.

Up to now, the photonic properties of triangular air lattices in silicon have been studied for perfectly circular structures, varying the size of the pores. Now, the shape of the columns will be generalized to an elliptical geometry. In this context it is interesting to note that Baba [14] computed the photonic band structures of various shapes of optical atoms for triangular photonic crystals. The lattices were assumed to be built up of circles, hexagons, squares and triangles, with only the lattices of spherical holes showing notable complete band gaps (see also $[15,16]$ ).

For simulating the experimental process of electrochemical pore formation, it is useful, to distinguish two boundary cases of elliptical pore shape variations $a_{e} / b_{e}$ :

(i) the air filling ratio is constant ( $\Rightarrow$ all pores have the same area, plausible etching mode),

(ii) the major axis of the ellipse is constant ( $\Rightarrow$ minor axis variations decrease the pore area).

To study the influence of deteriorating column roundness, we will focus on cell arrangements slightly below the maximum band gap arrangement. In this way it is possible to design elliptical "atoms" without overlapping, even in the case of higher eccentricity. The underlying model, and the notation used are illustrated in Fig. 1. First results have been discussed in $[12,13]$.

Starting at circular pores $\left(a_{e} / b_{e}=1.0\right)$ of the radius of $r / a=0.45$ the orientation $\varphi$ of the ellipses in the Brillouin zone and the eccentricity $a_{e} / b_{e}$ are systematically varied in Fig. 4 . The air filling ratio has been kept constant at the level of $\gamma_{e}=0.7346$ during the shape variations (case $i$ ). To identify the minimum complete band gaps, TE and TM band structures are calculated within half of the Brillouin zone, because of the breakdown of symmetry in the case of arbitrarily oriented ellipses. It turned out that the size of the complete gaps showed a $60^{\circ}$ angular oscillation period. The variations of the size of the band gap are minor, if the eccentricity is small $\left(a_{e} / b_{e}>0.90\right.$, back of Fig. 4). If the $a_{e}$-axis of the ellipse has an angle of $\varphi=30^{\circ}$ in the lattice, the gap size decreases up to $15 \%$ for an eccentricity of $a_{e} / b_{e}=0.8$ (see the dark front zone of Fig. 4). Then, the band gap is in the frequency interval $\Delta f \in\left[0.088\left(\varphi=30^{\circ}\right), 0.111\left(\varphi=7.5^{\circ}\right)\right]$, showing considerable angular variations, essentially tending to a decrease of the gaps. The study of randomly arranged elliptical pores in 2D photonic crystals will be subject of future work.

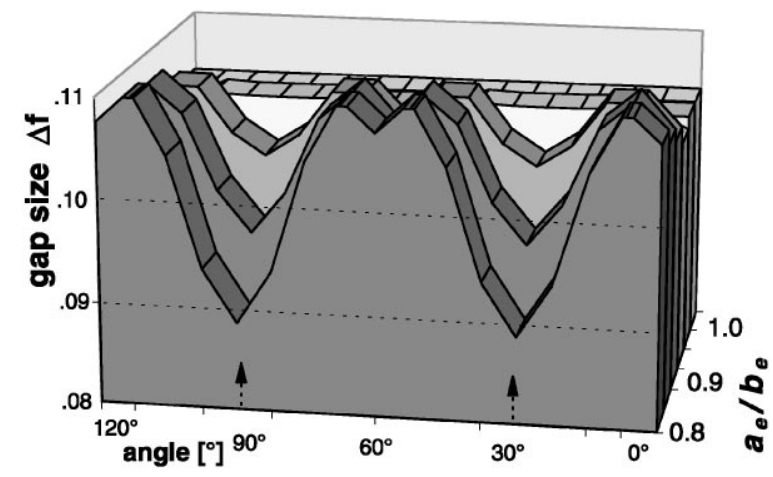

Fig. 4. Variation of the complete gap size $\Delta f[\omega a / \pi c]$ of a triangular lattice vs. the eccentricity $a_{e} / b_{e}$ of the pores and the ellipse orientation angle $\varphi$ in the lattice. Air filling factor $\gamma_{e}=0.7346$ constant (535 waves).
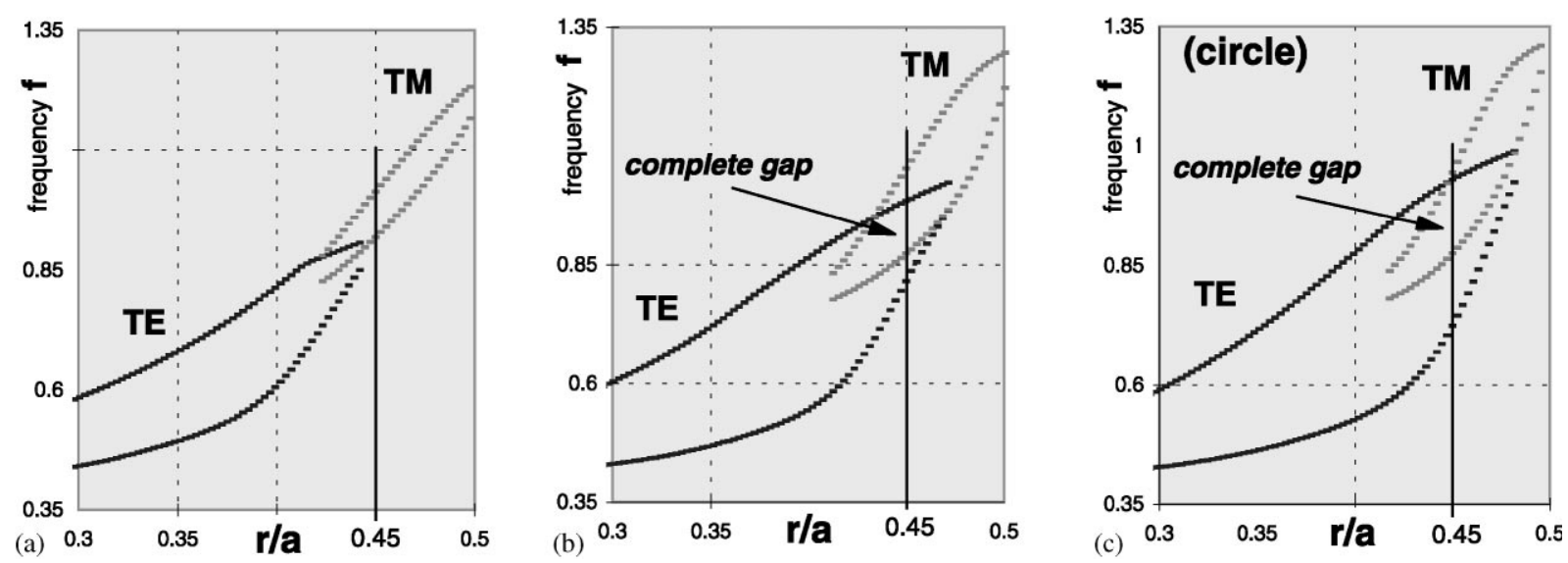

Fig. 5. Gap maps for triangular air lattices in Si ( $f[\omega a / \pi c], \varepsilon=11.7)$ : (a) ellipse $a_{e} / b_{e}=0.6$; (b) ellipse $a_{e} / b_{e}=0.8$; (c) ellipse $a_{e} / b_{e}=1$ (circle). 
If the major axis $b_{e}$ of the ellipse is kept constant and solely $a_{e}$ controls the eccentricity (case ii) the decrease of the gap size is a combined effect of falling filling factor and roundness changes. The computed series, not illustrated here, have revealed that the size of the remaining complete band gap basically decreases, with the roundness of the holes deteriorating.

Now, we consider a triangular air lattice in $\mathrm{Si}$, where the air filling factor and the orientation of the ellipse are kept constant while increasing the eccentricity $a_{e} / b_{e}$ $\left(\gamma=0.7346\right.$, corresponds to $\left.r_{\text {circle }}=0.45 a\right)$. Fig. 5 presents gap maps (cf. [6]) to illustrate the dispersion of the photonic crystals in a more comprehensive way. For the given lattice type and dielectric constant the locations of the band gaps are plotted for all pore radii practically relevant. The $x$-axis are scaled by the $r / a-$ ratio of circular holes having the same filling factor. The gap map of circular pores (cf. Fig. 2) is presented at the right-hand side. It should be noted that the number of included plane waves is limited to $2 \times 97$ up to now, because of the substantial set of band structure computations behind Fig. 5. It has been checked that the scaling behaviour of selected series of parameter variations holds, if the number of waves is enlarged step by step.

The combination and overlap of both the TM and TE band structures in one plot clearly reveal the existence of complete gaps with varying location and size. In Fig. 5, three selected eccentricities (left: $e=0.6$, middle: $e=0.8$ right: $e=1.0$ ) are chosen for an orientation angle $\varphi=0$ of the ellipse. It is obvious that strong eccentricity excludes complete photonic band gaps (left), because there are no more isolated non-overlapping 'photonic atoms'.

\section{Conclusions}

The paper describes theoretical studies of macroporous Si-based photonic crystals with respect to the corresponding dispersion behaviour. In particular, variations in the roundness of the 'photonic atoms' in the 2D lattice and their correlation with the photonic properties are quantified. The formalism derived analytically for elliptical columns has been applied to triangular lattices of air holes.

For geometrical reasons, the triangular lattice has a large filling ratio for increasing $r / a$, which limits the maximum possible eccentricity of the ellipses. On the basis of the implemented algorithm it is possible to conclude roundness quality criteria by varying the eccentricity of the air columns. In this paper, the pores have been varied conserving the air filling factor. The remaining size of the complete gaps can be interpreted in terms of the band gaps arising for fully circular arrangements. Elliptical pores in a triangular lattice show a $60^{\circ}$ angular period of complete gap size variations. The angular dependence of the band gap modifications is weak, for the eccentricity $0.9 \leqslant a_{e} / b_{e} \leqslant 1.0$. For an eccentricity of $a_{e} / b_{e}=0.8$, the gap variations reach $15 \%$ compared to circular pores.

\section{Acknowledgements}

The authors gratefully acknowledge the stimulating discussions with the co-workers of the porous materials group at the MPI Halle. Many thanks are due to P. Rennert for his encouraging interest in these theoretical studies.

\section{References}

[1] Yablonovitch E, Gmitter TJ. Phys Rev Lett 1989;63: 1950-3.

[2] John S. Phys Rev Lett 1987;58:2486-9.

[3] Grüning U, Lehmann V, Ottow S, Busch K. Appl Phys Lett 1996;68:747-9.

[4] Birner A, Grüning U, Ottow S, Schneider A, Müller F, Lehmann V, Föll H, Gösele U. Phys Stat Sol A 1998;165:111-7.

[5] Birner A, Schneider A, Müller F, Lehmann V. Annual Report Max-Planck-Institut für Mikrostrukturphysik, 1997. p. 30-1.

[6] Joannopoulos JD, Meade RD, Winn JN. Photonic crystals. Princeton, NJ: Princeton University Press, 1995.

[7] Plihal M, Maradudin AA. Phys Rev B 1991;44:8565-70.

[8] Meade RD, Rappe AM, Brommer KD, Joannopoulos JD. Phys Rev B 1993;48:8434-7.

[9] Bell PM, Pendry JB, Moreno LM, Ward AJ. Comput Physics Commun 1995;85:306-22.

[10] Ohtaka K, Ueta T, Amemiya K. Phys Rev B 1998; 57:2550-68.

[11] Sözüer HS, Haus JW, Inguva R. Phys Rev B 1992; 45:13962-72.

[12] Hergert W, Hillebrand R. Conference on Computational Physics, Granada, 1998. In: Garrido PL, Marro J, editors. Europhysics conference abstracts, vol. 22F. 1998. p. 276.

[13] Hillebrand R, Harms W, Hergert W, Birner A, Müller F, Annual Report Max-Planck-Institut für Mikrostrukturphysik, 1998. pp. 34-5.

[14] Baba T, Matzukaki T. Jpn J Appl Phys 1995;34:4496-8.

[15] Yablonovitch E, Gmitter TJ. Phys Rev Lett 1991;67: 2295-8.

[16] Villeneuve PR, Piche M. Phys Rev B 1992;46:4973-5. 